\title{
Role of Total Knee Arthroplasty in Different Joint Diseases- A Retrospective Study
}

\author{
Dr. Intikab Alam ${ }^{1}$; Dr. Anoop Suresh ${ }^{2}$ \\ ${ }^{1}$ Assistant Professor, Department Of Orthopaedics Yenepoya Medical College Hospital University Road, \\ Deralakatte Mangalore Karnataka, India \\ ${ }^{2} J u n i o r$ Resident, Department Of Orthopaedics Yenepoya Medical College Hospital \\ University Road, Deralakatte Mangalore Karnataka, India
}

\begin{abstract}
:
Aims:

1. To study the indication of TKA in our setup.

2. To evaluate the results clinically by assessing the functional improvement of the patients with reference to pain, walking ability and range of motion.

3. To study the implications associated with this procedure

Materials and Methods: We are reporting the retrospective study and the early results of 28 knees of Total condylar knee arthroplasty using either with or without posterior stabilizing designs. 28 knees in 25 patients who visited the department of orthopaedics at Bowring and Lady Curzon hospitals. Bangalore Medical college. Bangalore, during the year 2001 - 2003. Have been selected with appropriate indications. The patients with severe knee arthritis and intractable pain were screened consecutively to select the patients.

The patients with serious medical illness, infected arthritis, haemophilic arthropathy, charcoat arthropathy, and extreme complex deformed knees and follow up less than 6 months were excluded. Of the selected patients, 10 were $R A, 14$, were $O A$, and 1 had septic arthritis sequelae.
\end{abstract}

Results: There were $19(76 \%)$ females and 6(24\%) males. Right knee predominates (14-56\%) than left (8-32\%) and bilateral (3-12\%). The unilateral replacement did pose a problem in clinical evaluation and may have an effect on long term results. As two cases could not be followed up after 6months, evaluation was done for 26 knees in 23 patients. Pre-operatively all of our patients had moderate to severe pain and $38.5 \%$ had rest pain. Post-operatively only $19 \%$ had mild pain. Pre-operatively there were no excellent knees .11.6\% of the knees scored good, $26.9 \%$ scored fair and 61.5\% scored poor.

Post-operatively 65\% of the knees scored excellent, $16 \%$ good and 19\% scored fair

Conclusions: Our study of total condylar prosthesis series demonstrates that total condylar prosthesis is reliable, provide pain relief, average 90-95 motion and aood function.

Keywords: Knee;Arthritis;Total Knee Replacement;Physiotherapy

\section{Introduction}

Chronic knee pathologies are on a rise in our society in parallel to the rise in the expectancy over decades. Now it has become a common problem. Leaving many of our senior citizens crippled affection their activities of daily living severely. This has forced the orthopaedic surgeons to intervene at some point in the natural history of disease with a goal to achieve painless stable joints many of these individuals eventually require joint reconstruction surgery as very few are willing to accept to function limitation imposed by arthrodesis of the knee. Hence the role of TKA increased in recent times.

The goals of Arthroplasty included pain relief improved range of motion joint stability and enhanced function. Improvement in prosthetic design, precise surgical technique accurate instrumentation have yielded consistency high success rates in meeting the basic necessities of active life in both short and long term studies.

Investigations have developed standard evaluation methods for easy comparison of result worldwide.

Knee society developed by consensus a new rating system in which separate scales for knee rating in function assessment are used. The choice of ideal outcome measures in assess knee replacement remain a complex issue. For example should one be putting more emphasis on the patients overall improved well being and should more emphasis be placed on the technical success of surgery. Similar one should address the efficacy of the surgical procedure in terms of getting patients back to work or in attaining social independence. The present study emphasis of short term analysis of TKA using the total condylar prosthesis.

\section{Aims Of The Study}

1. To study the indication of TKA in our setup. 
2. To evaluate the results clinically by assessing the functional improvement of the patients with reference to pain, walking ability and range of motion.

3. To study the implications associated with this procedure

\section{Evolution of Total Knee Arthroplasty 7 '}

\section{Review Of Literature}

The parsian, jules p'eau (1830-1898), defined an arthroplasty as the creation of an artificial joint for the purpose of restoring motion. "The incongruity of the opposing joint surfaces with the additional complexity of the patellofemoral articulation have presented a challenge to reconstruct the joint for over century to the surgeons. The approach adopted toward restoration of function was as much influenced by current technology as by imagination.

\section{Resection and interposition arthroplasty ${ }^{8}$}

In 1860 Verneuil suggested using the soft tissue interposed between the raw bone ends after joint resection, later in 1863 he used joint capsule as the tissue for interposition. Fergusson 1861 described a case in which he resected the knee with creation of pseudoarthrosis which he reported as "a useful limb", but his results were compromised by shortening and ankylosis. Helferich 1894 reported success in treating ankylosed knee by using muscle for interposition. J.B Murphy 1905 conceded in his article that "the knee is the most difficult joint to secure perfect restoration of function". Various materials tried later are chromaticized pig's I bladder (Baer 191, Campbell 1921) fascial flaps (Murphy 1905) fat (Mac Ausland 1933) Nylon membrane (Kuhn \& Potter 1950). The results were compromised by instability or stiffness so leading the way for further work on it.

\section{Endoprostheses}

Initial attempts at knee replacement with endoprosthesis involved resurfacing procedures for the femur or the tibia. Drawing from the experience of Smith Peterson cup arthroplasty of the hip, Willis. Campbell(1940) designed a titanium prosthesis for the distal end of femur, after early satisfactory result procedure was abandoned because of poor results. Kraft and Levinthal (1954) also used acrylic prosthesis. Jones et al (1967) added intramedullary stem to vitallium prosthesis, a membrane routinely formed at the interface, which tended to be erosive painful in some cases.

Beginning in 1950, replacement prosthesis for the tibial plateaus were described by Depalma (1954), and fixed in place by McKveer's (1955) bicondylar plate fixed by flange, Macintosh by surface irregularities (1958), and Townley's by screws (1964), in spite of optimistic early reports, the frequency of complications and the emergence of semi constrained designs spelled the end of resurfacing arthroplasty by early 1970's.

\section{Hinge prosthesis ${ }^{8,9,10}$}

The general dissatisfaction with the results of earlier prosthesis, emphasis is laid on stability, adequate fixation which made Walladius $(1975,1960)$ to describe hinged acrylic prosthesis later modified to be constructed with vitallium the linked characteristic of the prosthesis made loss cruciate ligaments of no significance. Modifications of this prosthesis were developed by Shiers $(1954,1961)$, Young $(1963,1965)$, who relied on long intramedullary stem for stability. By contrast Guepar (1973) hinge was used with cement and it had articular surface for patella with the axis of hinge location posterior \& superiorly to simulate tibial "rollback" during flexion. But early enthusiasm with this design faded fast because of the reports on loosening, \& inability to absorb rotational stresses about long axis of the limb constituted an insurmountable flaw.

\section{Cemented Endoprosthesis ${ }^{8,10}$}

Gunston $(1971,1973)$ receives credit for describing a nonconstrained knee arthroplasty with cemented prosthesis. This polycentric design wide acceptance was inhibited by the complexity of surgery. The refinement of this concept were, the Marmor (1973) Unicondylar, Sledge and Manchester (Shaw and Chatterlee 1978), To. overcome the difficulty of orienting four components femoral \& tibial components were linked with strut respectively. The Geometric knee (Conventry et al 1970, Skolnich et al 1976) emerged as most popular design as it allowed for retention of cruciates.

The UCl prosthesis was an improvisation to accommodate rotation about long axis of the leg. Freeman and Swanson coincidentally developed the ICLH prosthesis which required sacrifice of the cruciates thus simplifying insertion. The first prosthesis incorporating a metallic tibial tray with interchangeable polyethylene inserts was introduced is the variable axis prosthesis in 1974. Good fellow (1978) introduced the concept of moveable plastic inserts designed to move posteriorly with flexion. This concept is now remerged as low contact stress (LCS). 
Insall et al $(1976,1979)$ made break through with evolution of "Total Condylar" configuration. In contrast to Duopatellar prosthesis which was intended to preserve posterior cruciate which later evolved into Kinematic I and II and Press - Fit condylar.

Cementless design series of prosthesis were Porous coated I anatomic II, the Miller-Galante, Tricon M, Genesis and Ortholoc.

Patellar resurfacing was introduced as a possible solution for patello femoral symptoms, but early enthusiasm was tempered by concerns about implant loosening, patellar fracture, a vascular necrosis and patellar dislocation (Insall et al 1982).

With continuous work in the field of designing newer prostheses, attempts have been made to imitate more closely the normal biomechanics of the knee joint and to take into consideration the patello femoral joint. At the same time, investigation of alternative implant fixation methods has achieved considerable advances. improvement in the design of components and in surgical and fixation technique has improved the outcome of the TKA.

\section{Indication And Contraindication}

The ideal candidate for surgery has moderate to severe pain that will be present during routine daily activities. Painful knee block to both full extension as well as full flexion. However, before surgery conservative measure should be tried including weight loss.

Indication :

$>$ Osteoarthitis

$>$ Rhematoid Arthritis

$>$ Osteonecrosis

$>$ Psoariasis, Gout with severe pain

\section{Contraindication :}

$>$ Patients with sepsis in and around knee

$>$ Traumatic conditions

$>$ Ankylosed knee

$>$ Extensor mechanism discontinuity and severe dysfunction

$>$ Recurvatum deformity secondary to muscular weakness.

\section{Relative Contraindication;}

These are numerous to mention few are,

1. Medical condition that can compromise the ability to withstand the risk of anaesthesia \& surgery, later rehabilitation protocol.

2. Atherosclerosis of the operative limb

3. Skin condition at operative site

4. Repeated UTI.

\section{Surgical Technique}

The primary indication for TKR is to relieve pain caused by severe arthritis with or without significant deformity. Patients ideally I suited for this procedure are elderly, more than 65 years with modest functional demands, with failed non-operative treatment and are not candidates for alternate procedures. However, it is also indicated in younger patients with multiple joint arthritis with limited functions.

Pre Operative Work up: Preparation of the patient was started 72 hours before surgery, with sending patients operative site skin swab \& urine for C/S. Patient's operative limb was painted with the MICROSHIELD (povidine iodine) allowed to dry in air \& wrapped the area with sterile cotton bandage for every 24 hours this was repeated.

Preoperatively parenteral antibiotics as started 30 minutes before the procedure \& continues for five days after surgery. Antithrombotic prophylaxis was given only when indicated.

Surgical approaches: Anterior midline incision is the most common incision used. If multiple incision scars are present the most lateral should be used because the blood supply to the skin of the anterior knee tends to come predominantly from medial side.

\section{Post Operative Protocol:}


Immediate post op patient was put on pain clinic analgesia for $24-48 \mathrm{hrs}$. Below knee skin traction was applied over the compression dressing, if deformity correction is exceeds 20 degrees.

From $2^{\text {nd }}$ post operative day static quadriceps exercises were started on $5^{\text {th }}$ post operative day wound inspection with aseptic precaution done knee mobilization started immediately after removal of the drains, PWB ambulation was allowed as patient tolerates pain. Suture removal on $12^{\text {th }}$ and $14^{\text {th }}$ days of post operative respectively.

\section{Follow up}

$1^{\text {st }}$ follow-up at 6 weeks

At interval of 3 months to intervals of 6 months

FWB is usually started at 1st follow-up in uncomplicated cases. At each follow-up patient was assessed as per the proforma. At the follow-up the surgeon and the observer assessed him.

\section{Materials}

We are reporting the retrospective study and the early results of 28 knees of Total condylar knee arthroplasty using either with or without posterior stabilizing designs. 28 knees in 25 patients who visited the department of orthopaedics at Bowring and Lady Curzon hospitals. Bangalore Medical college. Bangalore, during the year $2001-2003$. Have been selected with appropriate indications. The patients with severe knee arthritis and intractable pain were screened consecutively to select the patients.

The patients with serious medical illness, infected arthritis, haemophilic arthropathy, charcoat arthropathy, and extreme complex deformed knees and follow up less than 6 months were excluded. Of the selected patients, 10 were RA, 14, were OA, and 1 had septic arthritis sequelae.

\section{Methods}

We have designed a protocol of patient evaluation, management and follow up for documentation. All the patients were subjected to meticulous pre operative clinical, functional, and radiological evaluation. Detailed heamotological workup including bloodand urine culture was done. Thorough examination was done to rule out any focus of infection.

The clinical and functional evaluation parameters included pain, walking distance, range of motion, stability deformity extension lag, stair climbing, transfer walking aid, and muscle strength. The radiological evaluation parameters included alignment. Tibiofemoral defects and postoperative radiolucency.

Design selection was done appropriate to the individual joint surgery was performed under combined spinal and epidural was positioned in flexion. A broad spectrum antibiotic like cefaperazone, sulbactum combination of $1 \mathrm{gm}$ IV was given before tourniquet application. A thorough scrub was given. The part was painted with betadine and spirit. Sterile stockinet was used to drape the limb exposing only the operating area steri drape was used for operative site.

A standard midline approach with knee in flexion was used deeper anteromedial dissection was followed for arthrotomy. In two patients knee could not be flexed due to extension contracture. We used Insall Snip for patellar dislocation. Medial, lateral, posterior soft tissue release either minimal or extensive was done for soft tissue balancing and correction of deformities. Tibial and femoral osteophytes were excised. Tibial sectioning was done using extramedullary cutting jig with $8 \mathrm{~mm}$ section in 21 knees, and $10 \mathrm{~mm}$ in $7 \mathrm{knees}$. We have sacrificed both the cruciates in all knees. Femoral section was done with appropriate femoral rotation with reference to epicondylar line or whiteslide line 23 femurs were sectioned at 10mm, 4 knees at $13 \mathrm{~mm}$ and $1 \mathrm{knee}$ at $8 \mathrm{~mm}$.

The larger section was done in those patients where flexion deformity was very severe in or and How medica designs were used in 24 knees. 17 femoral and 13 tibial were of medium size, 7 femoral and 11 tibial were of small sized 3 posterior stabilized.

Next Gen design was used in difficult knees like extension contracture, mediolateral instability and larger tibial defects. 1PFC sigma posterior stabilizing was used in-patient with large posterior defect (size-2) in 2 patients we had the problem of anterior notching which was managed by fixation with bone grafts.

Tibial defects were managed by autologous posterior condylar grafts with screws.

The alignment and soft tissue balance was checked in extension and flexion 7 patellae were resurfaced using doom shaped all polyethylene patella. In 21 patients patella was only debulked. Trial components were assembled for proper fit and checked for soft tissue tension and balancing in flexion and extension. Patellar tracking was noticed normal in all except in 2 cases, which required lateral retinacular release. Cementing of components done by using one packet of bone cement. Tourniquet was released hemostasis was achieved by cauterization. Wound was closed in layers over suction drain. Antibiotics continued post operatively for one week. Standard postoperative protocol was followed to develop quadriceps to improve the range of motion and early weight bearing ambulation. Sutures removed at the end of two weeks. 
Immediate and follow up clinical radiological evaluation done at regular intervals. Final evaluation was done using KSS scoring system. All cases were photographically documented. The follow up period was from one month to one year ten months. The average follow-up period was 12.78 months. One patient was lost in the follow up because she died and months after surgery due to unrelated cause. The other patient who had infection undergone knee fusion also lost in follow up after six months by which time it has solidly fused. In effect the results are available for 26 knees in 23 patients.

Indications: 28 Knees

\section{Observations And Discussion}

\begin{tabular}{|l|c|c|}
\hline Rheumatoid Arthritis & 12 & $42.9 \%$ \\
\hline Osteo Arthritis & 15 & $53.6 \%$ \\
\hline Septic Arthritis Sequelae & 1 & $3.6 \%$ \\
\hline
\end{tabular}

Most of the indications in our study belong to OA \& RA group. This could be the same as in the literature published. Indications in RA \& OA differ substantially since the former condition is progressive and polyarticular. Management should be focused by treating the patient as a whole. Evaluation of these two different conditions by a common rating system may not appropriately reflect the results.

The walking ability, ROM, transfer were all significantly lower in RA than in OA.

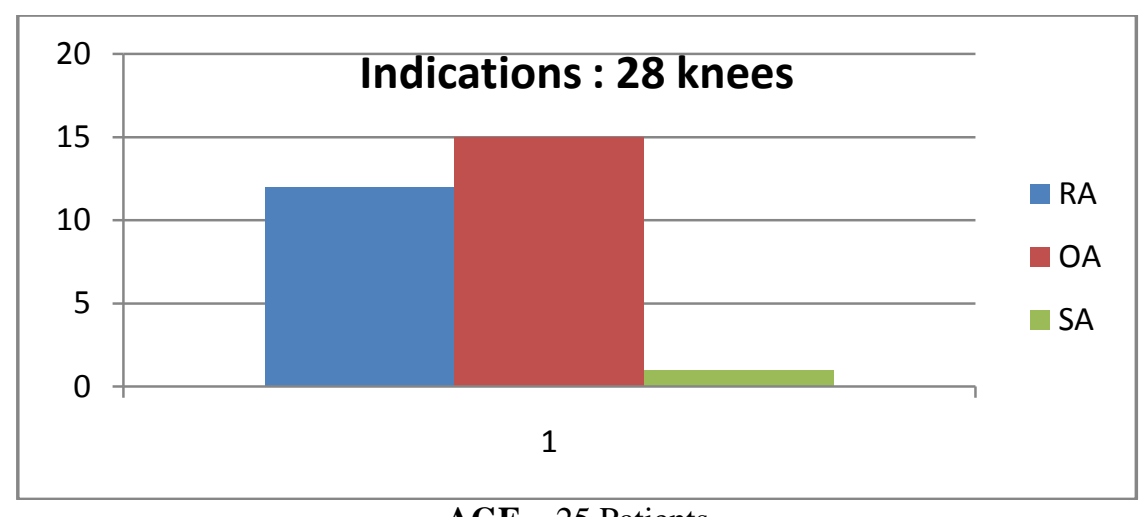

AGE - 25 Patients

\begin{tabular}{|l|l|l|}
\hline $25-34$ & 1 & $4 \%$ \\
\hline $35-44$ & 1 & $4 \%$ \\
\hline $45-54$ & 11 & $44 \%$ \\
\hline $55-64$ & 7 & $28 \%$ \\
\hline $65-74$ & 4 & $16 \%$ \\
\hline $75 \&>$ & 1 & $4 \%$ \\
\hline
\end{tabular}

Average age 53.2 years

The average age of 53.2 years in our study may be attributed in view of average lesser life expectancy of Indians

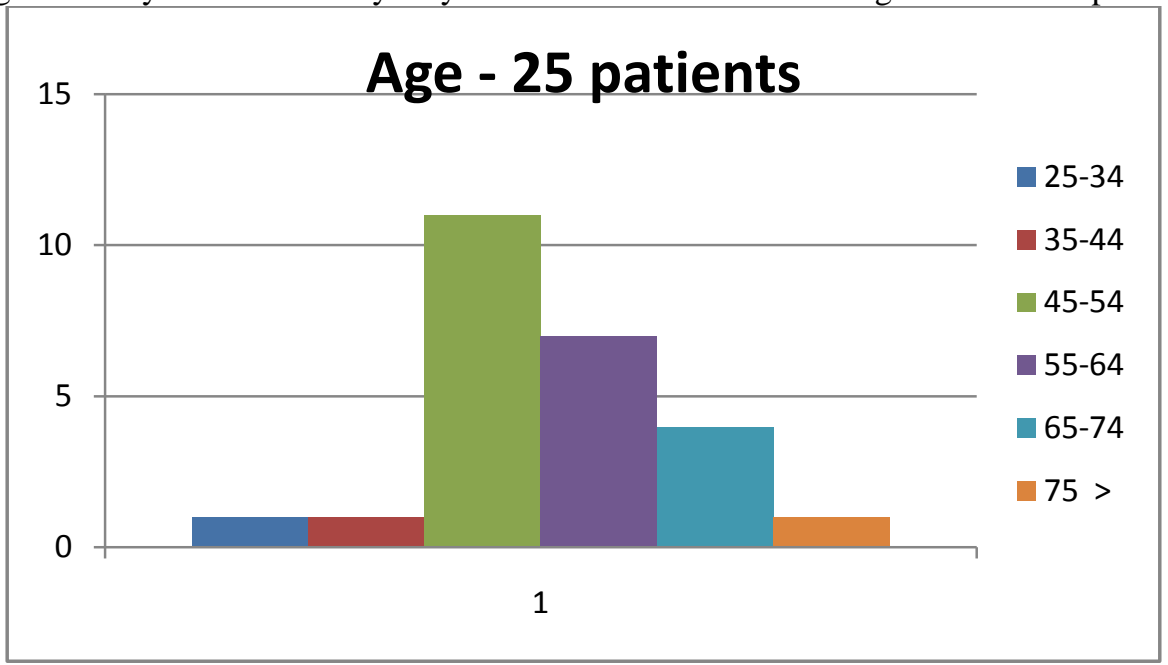


Insall $\mathrm{JN}$ et al has suggested that Joint replacement should be avoided in patients younger that $60 \mathrm{yrs}$ of age and that alternative surgical techniques such as high tibial osteotomy or Arthrodesis should be done whenever feasible.

The difference in outcomes in different age groups is thought to be involved in, among other things, activity level. In studies of knee biomechanics no correlation has been found between knee alignment angles and age40. However, younger patients with unsuccessful TKA result had OA more frequently than $\mathrm{RA}^{41}$.

Tankersley WS et $\mathrm{al}^{42}$ showed that total knee arthroplasty in very aged who were over 85 yrs of older, is safe and reliable procedure for management of pain and deformity secondary to Arthritis of knee however large number required, the continued use of Ambulatory aids after surgery and complication tend to be not greater than those of comparison groups of younger patients and their quality of life often is improved markedly.

SEX: No. of patients: 25

\begin{tabular}{|c|c|c|}
\hline Male & 6 & $24 \%$ \\
\hline Female & 19 & $76 \%$ \\
\hline
\end{tabular}

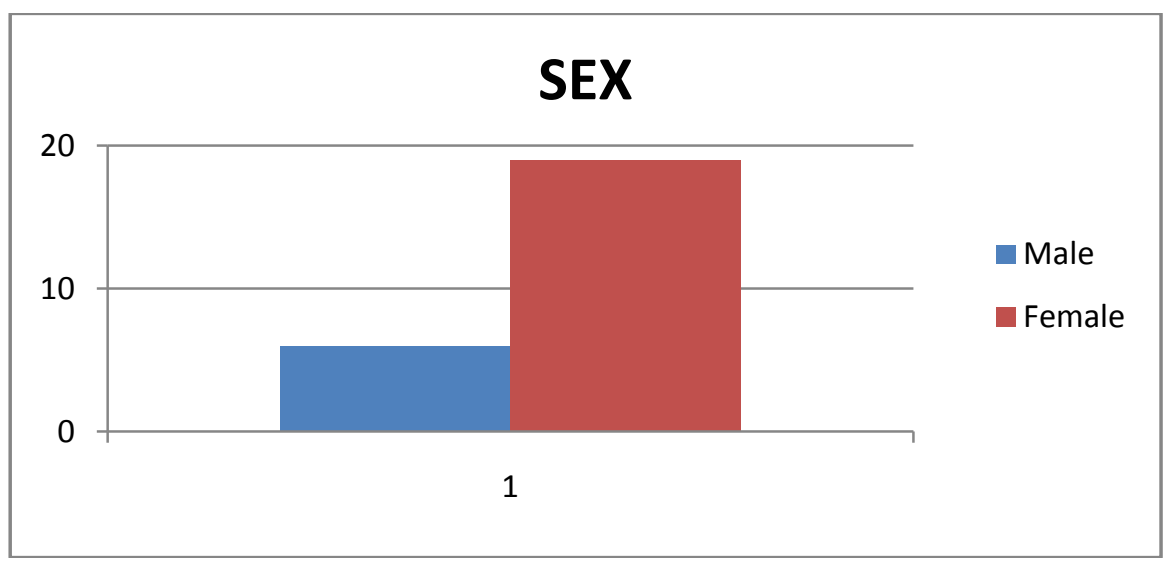

In our study male to female ratio is comparable to others.

Barrett et al ${ }^{43}$ noted differences in degenerated joint diseases between the sexes. Whereas the numbers of men and women with DJD varus were about equal, women with DJD valgus knees were twice as common as men, and primary patello femoral arthrothesis was 7 times more frequent in women. The explanation here lies in anatomical differences, namely the increased Q-angle in women. Also patellar complications have been seen to occur more commonly in young, heavy, male patients ${ }^{44}$. However, 3 multiple center studies report that neither such nor weight had any effect on the durability of TKA ${ }^{45,46}$.

SIDE No of patients: 25

\begin{tabular}{|l|l|l|}
\hline $\mathrm{R}$ & 14 & $56 \%$ \\
\hline $\mathrm{L}$ & 8 & $32 \%$ \\
\hline $\mathrm{B} / \mathrm{L}$ & 3 & $12 \%$ \\
\hline
\end{tabular}

Most of the patients in our study belong to OA and RA group who were generally either pausiarticular or polyarticular. Our bilateral procedures were much lesser than various reports published. This may have a bearing on the long-term survivorship of joints and may pose a problem in evaluation of functions.

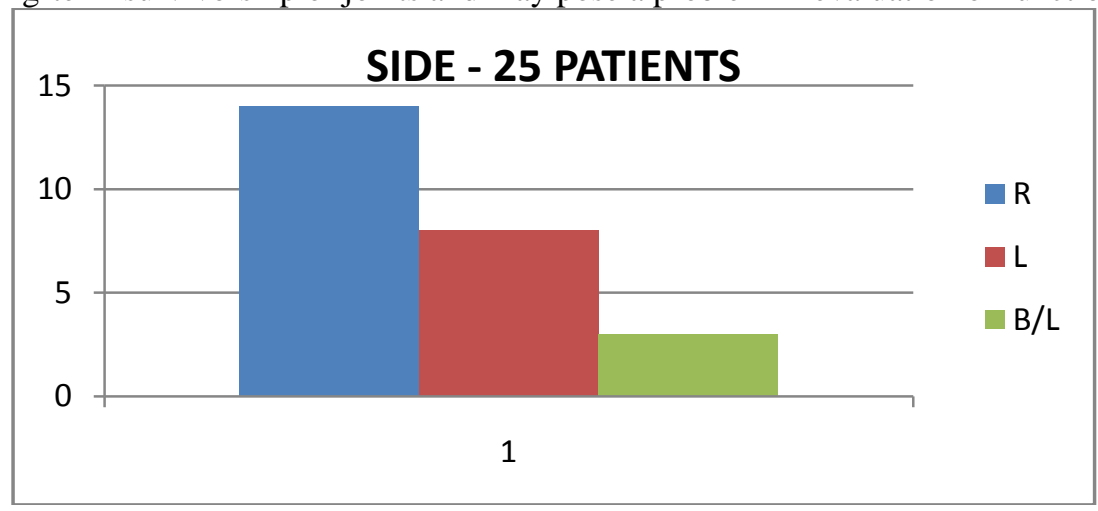


PAIN - 26 knees

In most of the cases the indication for TKR was chronic joint pain, moderate to serve, not responding to conservative treatment, with or without deformity. Pain is an important parameter not only as an indication for TKA, but also for the evaluation of the results,

Pain can be categorized into mild, moderate and severe based on the intensity, duration and response to medication. The pain parameter was given 50 points in KSS and 30 points in HSS. This indicates its significance. Pain has an influence on other parameters of functional evaluation, as it is associated with physical activity. Hence, alleviation of pain is the prime criteria of TKA.

Our study has shown that all the pre-operative moderate and severe pain knees become almost painless (80.8\%) or mildly painful (19.2\%) knees. All the 5 knees where there was mild pain belonged to RA group.

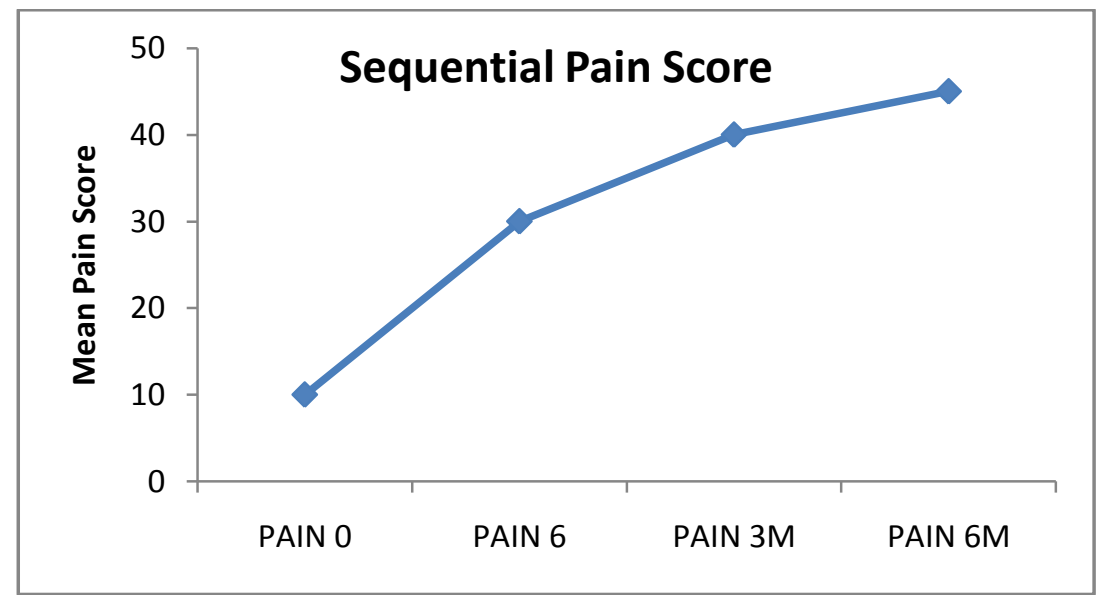

It has been estimated that $80 \%$ of individual over 55 yrs of age have degenerative arthritis of the knee and that $10 \%$ have pain, which limit acitivity ${ }^{53}$, It is not clear why some have more pain than others. Pain is a subjective sensation, the severity of which may not relate directly to the apparent cause. This variable response of pain is muiti factorial Neurogenic inflammation, measured in serum and joint fluid, has been postulated as one reason why some degenerative joints hurt and others do not .

Pre operatively, patients with the various alignments usually complain of pain, whereas those with the valgus or patello femoral disease describe the knee as a weak, unstable or deformed. Because pain is the most compelling reason for seeking medical intervention, patients with varus may seek evaluation earlier in the course of their disease.

Post operatively pain has been associated with infections loosening, reflex, sysmpathetic dystrophy. Ritter et $\mathrm{al}^{55}$ found no correlation between these abnormalities and pain. In the case of totally replacement, pain of the patellar origin is usually held as a distinct pain type, described as retro patellar or anterior knee pain.

\section{Range Of Movement (Rom) 26 Knees}

In general, although the principle movement of the knee is flexion and extension significant rotation and lateral movement also occurs. In the normal knee some posterior rollback of the femur occurs as the knee flexes, and the instantaneous center moves back and forward some mille meters. Brantigan and Voshell observed that both menisci slide backward with flexion, the medial meniscus moving only a few millimeters, the lateral atleast one centimeter. When measuring the load against the knee joint the tibial contact points on the medial side have been anterior in extension, and moved to center in flexion, to remain their further flexion. On the lateral side, the contact point is initially central and moves steadily posteriorly with flexion ${ }^{56,57}$

It has been demonstrated that $67^{\circ}$ of flexion of knee is required for swing phase of gait, 83 degrees to climb stairs; 90 degrees to descend stairs; and 93 degrees to rise from chair. Factors that influence the final range of motion after knee Arthroplasty, therefore, are significant determinants.

One of the primary goals of TKA is the restoration of a painless functional range of motion ${ }^{58}$. After arthroplasty, the range of motion can be adequate for good function at not the same as normal. Upto 113 degree of knee motion is needed for healthy adults to rise from a chair and some activities such as the lifting of an object from the floor may require 117 degree ". Hunger for and Krackow ${ }^{60}$ gave that an arc of 5 to 70-80 degrees as that usually providing normal gait, including stair walking. Other are of the opinion that flexion beyond 90 degrees is desirable to facilitate rising from a stair, and this is especially important for patients with bilateral knee disease". 
The most important determinant of post operative ROM is the range prevailing before arthroplasty $^{62,63,64}$. Patients with the pre operative flexion less than 75 degrees have shown an improvement and those with the pre operative flexion greater than 95 degrees a decrease in post operative flexion. ${ }^{58,65}$

Shurman et $\mathrm{al}^{66}$. Who stated that one could anticipate gains in flexion for up to one year, with the most significant gains occurring within the first three postoperative months. This was true for rheumatoid and osteroarthritic patients alike. Rorabeck et $\mathrm{al}^{67}$. Utilizing a variety of implant designs also found no significant change in flexion beyond one-year post arthroplasty. However, it was not influenced by the extent of disease (mono Articular in comparison with poly Articular) or by treatment with unilateral or bilateral procedure. Knees with pre operative flexion of $>100$ degree had lost flexion at follow up but those with $<100$ degree had gained flexion - This so called 'regression' toward the mean might indicate that there is some restricting factor such as prosthetic designs, insertion technique or post operative therapy, Thus advocated best time to correct flexion contrecturt is at time of surgery and during the in hospital post operative period, however, some patients may improve > 10 degree even after $1 \mathrm{yr}$.

A correlation has been ascertainment between diagnosis and postoperative range of motion ${ }^{58,68}$. A positive correlation was found between pre and postoperative flexion with stronger predictability in an inflammatory patient group (parsley et al 1992, Harvey et al 1993). Dennis et al $^{69}$ found, in contrast, no statistically significant differences between $\mathrm{OA}$ and RA in a comparison of the pre and postoperative flexion.

In clinical follow up studies of TKA, the average range of motion is usually between 80 and 120 degrees, depending on type of the prosthesis and the patient population. There is usually considerable variation between different series ${ }^{50,70}$. In summary, preoperative flexion does exert a significant influence on postoperative flexion. The relationship between preoperative flexion and postoperative flexion provides the surgeon with greater insight into the anticipated flexion following TKA. The patient can therefore be counseled with greater reliability on the expected results. The cemented status of the tibia does not influence these results when evaluated at one year, but further analysis may demonstrate differences such as those suggested by Rorabeck et al.

No correlation has been found between flexion and the age or the gender \& diagnosis of the patients, full extension is almost obtained by six months \& there is no change form 6-12 month $^{71}$.

Prosthetic component alignment effects ROM. if the femoral component is positioned in anterior tilt, cruciate ligament tension is reduced and flexion increased ${ }^{56,72}$. Tilting the tibia! component in the sagittal plane likewise effects flexion, a 10 degree posterior tilt gives 30 degrees of extra flexion and an anterior tilt the opposite effect. McAuley et al ${ }^{56,73}$ concluded stiff knee can be replaced. Success fully but results are inferior to those of a standard primary total knee arthroplasty.

There is no consensus on the matter of patellar resurfacing, but Murray ${ }^{74}$ established that flexion is increased between 5 and 10 degrees following patellar resurfacing.

Sequential Range of Motion

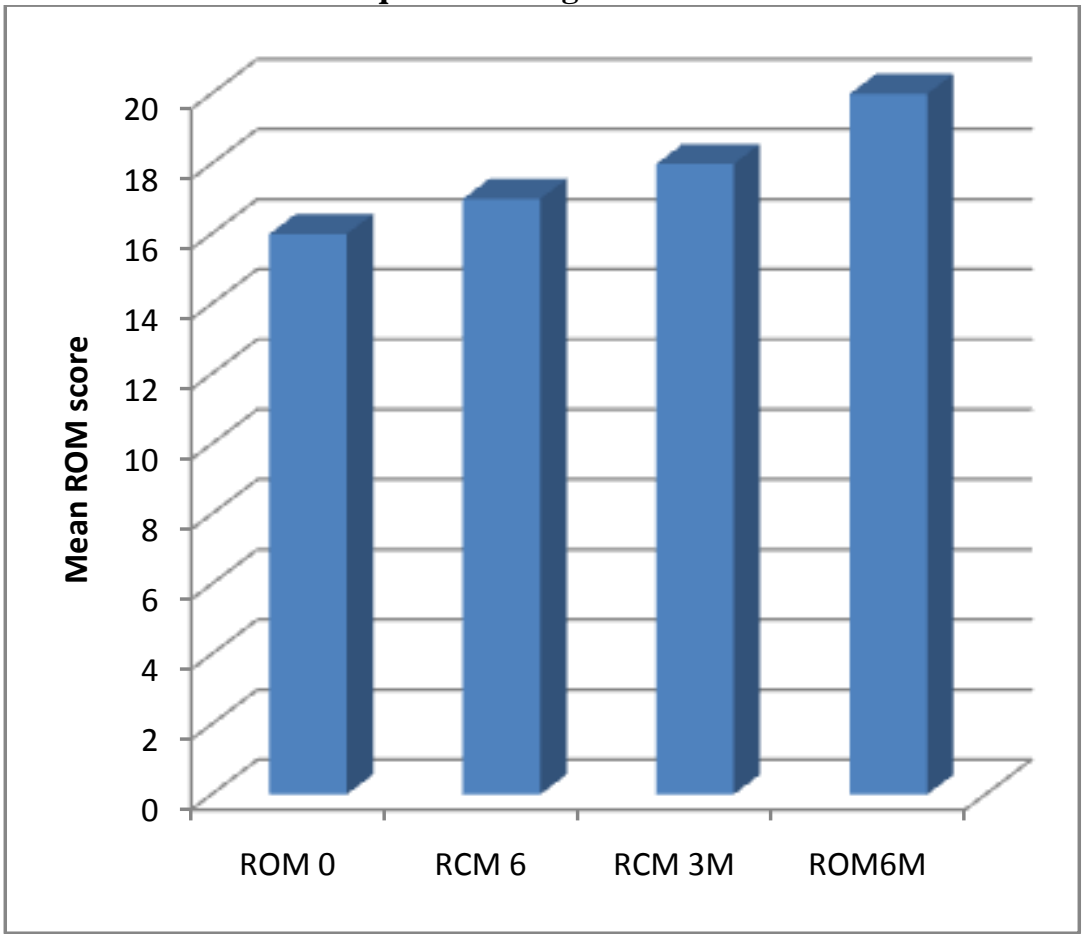


The ROM improved considerably with the average preop range of 75 degrees to 103 degrees on average at the last visit. Range didn't improve in three of the knees of which one was having the diagnosis of post septic arthritis.

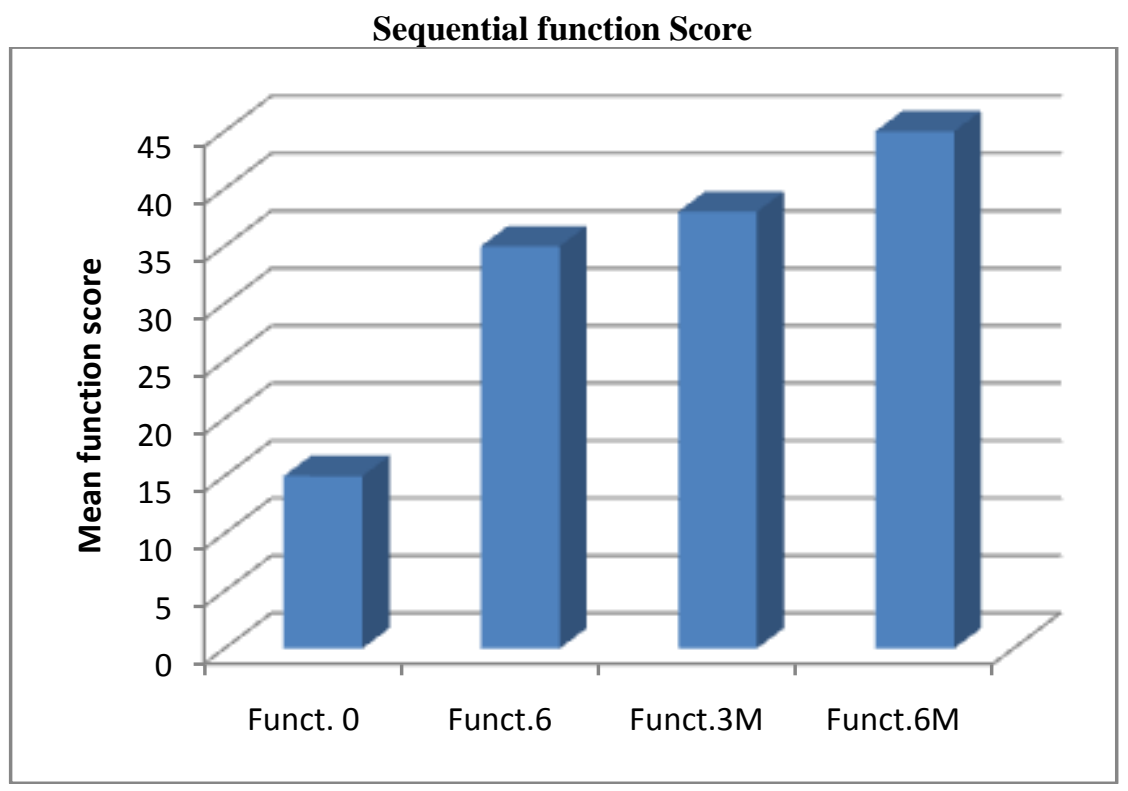

The average function score rose significantly at 6 months follow up. Preoperatively $(10.52 \%)$ were unable to walk and climb stairs. (71.05\%) were house bound and unable to climb stairs. (18.43\%) were able to walk less than 5 blocks. (78.9\%) were using walking aids.

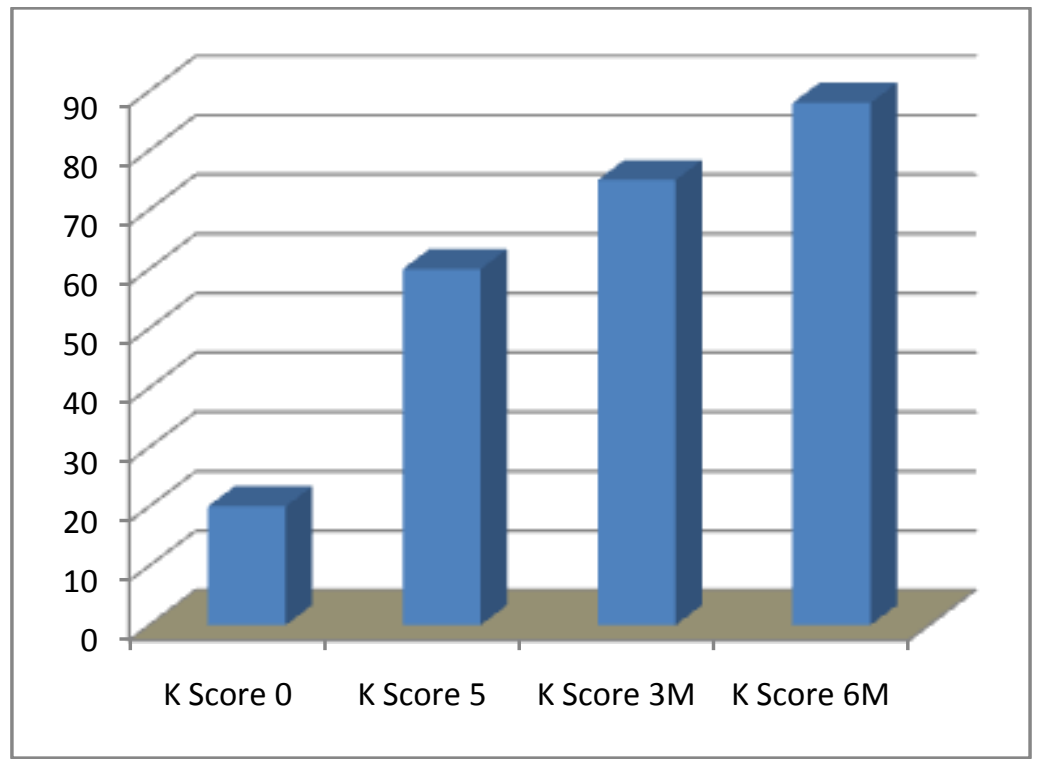
follow up.

The knee scores increased significantly from 24.3 points pre-operatively to 88.7 points at the last

\section{Summary}

We did Retrospective study of TKA in 28 knees in 25 patients during 2001-03, with RA12, OA15 and one septic arthritis sequelae. There were $19(76 \%)$ females and 6(24\%) males. Right knee predominates (14$56 \%)$ than left $(8-32 \%)$ and bilateral (3- 12\%). The unilateral replacement did pose a problem in clinical evaluation and may have an effect on long term results.

As two cases could not be followed up after 6months, evaluation was done for 26 knees in 23 patients.

Pre-operatively all of our patients had moderate to severe pain and $38.5 \%$ had rest pain. Postoperatively only $19 \%$ had mild pain. 


\section{These patients belong to RA group.}

Pre-operative $8.6 \%$ unlimited walkers increased to $73.9 \%$ postoperatively. There were no postoperative house bound and unable patients compared to pre-operative $35 \%$ of such patients.

Pre-operative average ROM of 75 improved to post-operative ROM of 103 . Pre-operatively $58 \%$ of our patients had compromised ROM of 5-60; no such cases were there postoperatively. 54\% had 61-90 ROM and 46\% had 91-120 ROM postoperatively. All of our patients had functional range of movements.

Pre-operatively $91 \%$ of patient needs walking aids \& post operatively only $26 \%$ used it.

Pre-operatively there were no normal stair climbers; post-operatively it was $65.2 \%$ (15). Similarly there were only $39 \%$ who could transfer themselves normally which increased to $82.6 \%$ post-operatively.

Pre-operatively there were no excellent knees .11.6\% of the knees scored well, $26.9 \%$ scored fair and $61.5 \%$ scored poor.

Post-operatively $65 \%$ of the knees scored excellent, $16 \%$ good and $19 \%$ scored fair

Radiological evaluation pre-operatively and post-operatively is one of the technically difficult aspects of TKA. Also, obtaining the correct or true view calls for real technical skills. Lack of facilities in obtaining a standing X-ray and full length X-rays must be kept in mind. In statistical evaluation, the above facts need emphasis.

Radiolucency was observed in zone 1 on tibial side only in two cases. Our period of study is too short to comment on this.

We had one knee, which was deeply infected which was salvaged with fusion. Another case had partial peroneal nerve injury, which did not hinder the functions much

\section{Conclusion}

$>$ Total knee replacement is an acceptable option for patients who have degenerative arthrosis that has not responded to non-operative treatment in improving the functional ability, although longer follow up could alter our conclusions.

$>$ Rating systems are influenced by numerous factors linked to patient's general health and condition.

$>$ Careful patients' selection must be done as young, active and heavy patients may abuse artificial knee.

$>$ Pain relief after total knee arthroplasty is remarkable and dramatic.

$>$ Preoperative flexion does exert a significant influence on postoperative flexion.

Our study of total condylar prosthesis series demonstrates that total condylar prosthesis is reliable; provide pain relief, average 90-95 motion and aood function.

\section{References}

[1]. Kreibich DN, Vaz M, Bourne RB, Rorabeck Ch, Kim P, Hardie R,. Kramer J, and Kirkley A, what is the best way of assessing outcome after total knee replacement? : CORR331:221 -225, 1996.

[2]. Wright JG, Feinstein AR: Improving the reliability of orthopaedic measurements, JBJS 74B: 287-291, 1992.

[3]. Callahan CM, Drake BG, Heck DA, Dittus RS: patient outcomes following tricompartmental total knee replacement,JAMA271: 1349-1357,1994

[4]. Install JN, Dorr LD, Scott RD, Scott WN: rationale of knee society clinical rating system CORR, 248: 13-14, 1989.

[5]. Gartland JJ: Orthopaedic clinical research. Deficiencies in experimental design and determinations of outcome, JBJS70 A: 13571364, 1988 .

[6]. Kellet RB, Rudicer SA, liang MH: outcomes research in Orthopaedics JBJS 75A: 1562-1574, 1993.

[7]. Shetty AA, Tindall Ating P and Healty FW: the evolation of total knee arthroplasty part I: introduction and first steps. Current orthopaedics 17:322-325,2003

[8]. Richaard S laskin (ED) : Total knee replacement, Springerverlag, NewYork, pp 3-38, 1991

[9]. Campbell's operative orthopaedics, Voi I Mosby, new York, pp 245 313, 2003.

[10]. Scott WN : The Knee, VoI II Mosaby, NewYork, pp 1045- 1103, 1994.

[11]. Nisell R: on the biomechanics of the knee. Acta OrthopScand 216:4-11, 1985.

[12]. Maquet P Mechanics of the nee. In: Biomechanics of the knee $2^{\text {nd }}$ ed. Springer - Verlag, New York, pp $9-741984 \mathrm{a}$.

[13]. Bullough P, Goodfellow $\mathbf{J}:$ the significance of the fine structure of the articular cartilage. JBJS 50B : 852 - 857, 1986

[14]. Grood Es, Suntag WJ, Noyes FR, bulter DL: Biomechanics of the knee- extenstion exercise, JBJS 66A: $725,1984$.

[15]. Hungerford DS, Barry M: Biomechanics of the patellofermoral contact pressures: the influence of Q- angle and tendfermoral contact, JBJS 66A :715, 1984.

[16]. Huberti HH, Hayes WC: Patellofermoral contact pressures: theinfluence of Q- angle and tendofermoral contact, JBJS 66A:715, 1984.

[17]. Maquet P (1984). The pathomechanics of osteoarthritis of the knee. In: Biomechanics of the knee, 2nd ed. Springerverlag, New York, pp $75-131$.

[18]. Stiehl JB,Komistek RD, Dennis DA, et al : Fluroscopic analysis of kinematics after posterior - cruciate - retaining knee arthroplasty, JBJS 77B: 884, 1995.

[19]. Ritter MA, Campbell E, Faris P, Keating EM: Long - term survival analysis of the posterior cruciate condylar total knee arthroplasty: a 10- year evaluation, J Arthroplaaty 4: 293, 1989.

[20]. Ritter MA, herbst SA, Keating EM, et al: Long - term survival analysis of a posterior cruciate - retaining total condylar total knee arthroplasty, clin Orthp 309; 136, 1994.

[21]. Andriacchi TP, Galantt JO: retention of the posterior cruciate in total knee arthroplasty, J Arthroplasty 3 (suppl) ; $13,1988$.

[22]. Mahoney OM, Noble PC, Rhoads DD, et al: posterior cruciate function following total knee arthroplasty, J arthroplasty 9: 569,1994 . 
[23]. Faris PM, herbist SA, Ritter MA, Keating EM: the effect of preoperative knee deformity on the initial results of cruciate -retaining total knee Arthroplasty, J Arthroplasty 7:527, 1992.

[24]. Pagano De, Stern SH, Insall JN: Revision total arthroplasty for failed unicompartmental replacement, J Bone Joint Surg 73A:176, 1998.

[25]. Scott RD, Thornhill Ts: Posterior cruciate supplementing total knee replacement using conforming inserts and cruciate recession: effect on range of motion and radiolucent lines, clinOrthop 309: 146, 1994.

[26]. Scott RD: Primary knee arthrolpasty: long - term outcomes and expectations - cruciate - sparing total knee arthroplasty,orthopedics 17: 839,1994

[27]. Scott Wn, rubinstein M, Scuderi G: results after knee replacement with a posterior cruciate - substituting prosthesis,JBJS70 A: 1163,1988

[28]. Bartel D, Burstein A, santavicca E, Insall J: performance of the tibial components in total knee replacement, J. Bone JointSurg (AM) 64: 1026 -1033, 1982,

[29]. Bartel DL, Bicknell VL, weight TM: the effect conformity, thickness, and material on stress in ultra high molecular weight components for total joint replacement, J bone Joint Surg 68A: 1041, 1986.

[30]. Tsao S, Mintz L, McRae Cr, et al: failure of the porous- coated anatomic prosthesis in total knee arthroplasty due to severe polyethylene wear, J Bone Joint Surg 75A: 19, 1993.

[31]. Scuderi GR, Insall JN, Windsor RE, Moan MC: Survivorship of cemented knee replacements, J bone Joint Surg71B:798,1989.

[32]. Aglietti P. Buzzi R, De Felice R, Giron F: the Insall-Burste in total knee replacement in osteoarthritis: a 10 year minimum followup, J arthroplasty 14:560, 1999.

[33]. Rand JA: Comparison of metal - backed and all - polyethylene tibial components

[34]. in cruciate condylar total knee arthroplasty. Jarthroplasty 8: 307, 1993.

[35]. Callihan SM ans hally DK: Prospective analysis of Sheehan total knee arthrplasty. CORR 192: 124 - $131,1985$.

[36]. Freeman MAR, Swanson SAV and Todd RC: Total replacement of the knee

[37]. design considerations and early clinical results. Artao Orthop Belg 39: 181 -202, 1973

[38]. Kettle kamp DB and Thompsom C: Development of a knee scoring scale,

[39]. CORR 107: $93-99,1975$.

[40]. Hungerford DS and Kinna RV : Preliminary experience with a total knee prosthesis with porous coating used without cement., CORR 176: 95 - 107, 1983

[41]. Bert J.M and maxchaka K,: the arthroscopic of unicompartmental gon arthrotis.

[42]. A five arthroplasty plus Arthoscopic debridmimt and year follow- up study of

[43]. Abrasion Arthroscopic debridment alone, Arthroscopy 5: 25- 32, 1989.

[44]. Timoney J.M knersl J.S, Barrack R.L and Alexander A.H: arthroscop update: 6Arthroscopy in the osteoarthritic knee long follow up. Orthop. Rev. 19: 371- 373, 376 - 379, 1990.

[45]. Hsu R WW, Himeno S, Coventry MB and Choo EYS (1990):normal axial alignment of the lower extremity and load- bearing distribution at the knee: CORR 255: 227, 1990.

[46]. Stern SH, Bowen MK, Insall JN nad Scuderi GR: Cemented total knee Arthroplasty for gonarthrosis in patients 5 yrs. Old or younger. CORR 260: $124-129,1990$.

[47]. Tan Kersely Ws, Hunger ford DS: Total knee Arthroplasty in the very aged, COR: $316: 45-46,1995$.

[48]. Barrett JP, Roshkoff E, Sirrna EC and Nilson A: Correlation of roentgenographic patterns and clinical manifestations of symptomatic with diopathic OA of the knee. CORR 253: 179-183, 1990.

[49]. Rosen berg AG, Andriacehi TP, Barden R and Galante JO. Patellar component

[50]. failure in cementless total knee Arthroplasty. CORR 236: 106-114, 1988.

[51]. Rand JA and II strup DA: survicorship analysis of total knee Arthroplasty: cumulative rates of survival of 9200 total knee Arthroplasties, JBJS 73A: 397 - 409, 1991.

[52]. Scuderi GR, Insall JN, Windsor RE and Moran MC; Survivership of cemented knee replacements, JBJS 71-B,789- 803, 1989.

[53]. Leach RE, Baumgard S and Broom J; obesity: its relationship to osteoarthritis Of the Knee, COR 93: 271- 273, 1973.

[54]. Matthews LS, Goldstein SA and Kaufer H. Experiences with three distinct types of total knee joint arthroplasty, CORR 192 :97107,1985

[55]. Ahlberg A and Lunden A ; secondary operations after knee joint replacement, CORR 156 :" 170 - 174,1981

[56]. Townley Co. the anatomic to6tal knee resurfacing Arthroplasty:CORR 192: 82- 96, 1985.

[57]. Windsor RE, Scuderi GR, Moran MC and insall JN; Mechanisms of failure of the femoral and tibial components in total knee arthroplasty. CORR $248: 15-20,1989$.

[58]. Hostick WB, Lotke PA, Baldwin A: total knee Arthroplasty in patients 80 years of age and older, CORR 299: 9-12, 1994.

[59]. Ke;;egre JH Lawrence JS: osteo Arthrosin and disc degeneration an urban population: Ann. Rheum. Dis 17: 388- 397, 1958.

[60]. Marshall K.W, Chiv B and Inman RD: Substance P and arthritis analysis of plasma and synovial fluid levels. Arthritis Rheum, 33: $87-90,1990$.

[61]. Ritter MA: Post operative pain after total knee Arthroplasty. J Arthroplasty 3: 337 - 339, 1997.

[62]. Walker PS and Gary A: range of motion on total knei Arthroplasty A computer analysis.CORR 262: 227=235, 1991

[63]. Nilsson KG, Karrhom J and Eke lund L: Knee motion in total Knee Arthropiiiity. A roentgen stereo photo grammetric analysis of the kinematics of the Tricon, M Knee prosthesis. CORR 256: 147-161, 1990

[64]. Parsley BS, Engh GA and Dwyer KA: preoperative fiexion.. Does it influence post operative flexion after posteriorcruciateretaining total knee Arthroplasty?, CORR $275: 204$ - 210, 1992.

[65]. Laubenthal KN, Smidt GL and Kettle Kamp DB: A quantitative analysis of knee motion during activities of daily living. Physical therapy 52:34-43, 1972 .

[66]. Hungerford DS and Kracknow KA: Total joint Arthroplasty of the knee. CORR 192: 23 - 33, 1985

[67]. Friedman RJ, Hirist P, Poss R, Kelsery K and Sledge CB: results of revision total knee Arthroplasty performed for aseptic loosening. CORR 255: 235 - 241, 1990.

[68]. Insall JN, Hodd.RW, Flawn LB and Sullivan DJ: the total condylar knee prosthesis in gonarthrosis - A five to nine year follow - up of the first one hundred consecutive replacements. JBJS 65-A: 619 -628, 1983.

[69]. Ronawat CS, Insall JN and shine J: DUO condylar knee Arthroplasty, CORR 120: 76 - 82, 1976

[70]. Rorabeck CH, Bourne RB and Nott L: the cemented kintmatic - Ill and the non - cemented porous coated anatomic prosthesis for the total knee replacsmint, JBJS 70 - A: 483 - 490, 1983

[71]. Anouchi YS, Mc Shane M Kelly Jrn F, Elting J and Stichi J: Range of Motion. In total Knee replacement, CORR 331: 87 92,1996. 
[72]. Schurman DJ, Parker JN AND Ornstein D: Total Condylar protstesis. A study of factors influencing ROM as late as 2 yr after ARthroplasty, JBJS. 67 A, No.7; 1006-1014.

[73]. Rorabeck C.H. Bourne R.B, and Nott L: The cemented kinematic - II And the noncemented porous coated anatomic prosthesis for the total knee replacement.JBJS 70A: 483,1983.

[74]. Harvey IA, Barry K, Kirby SPJ, Johnson R and Elloy MA Factors affecting the range of movement of total knee arthroplasty, JBJS 75-B: 950-955, 1993.

[75]. Dennis DA, Clayton ML, O' Donnell S, Mack RP and Stringer EA: Posterior cruciate condylar total knee arthroplasty. Average 11year follow-up evaluation, CORR 251: 168-176, 1992.

[76]. Ranawat CS and Boachie - Adjie O: Survivorship analysis and results of total condylar knee arthroplasty. Eight to eleven year follow-up period, CORR 226: 6-13, 1988.

[77]. Ritter MA and Stringer EA: Predictive range of motion after total knee replacement. CORR 143:115-119, 1979.

[78]. Kaufer H and Mattkews LS: Sphero centric knee arthropasty. CORR 145: 110-116, 1979.

[79]. McAuley JP, Harrer MF, Ammeen D and Enger GA: Outcome of knee ARthroplasty in patients with poopreoperative ROM: CORR 404: 203 207, 2002

[80]. Murray DG Total knee arthroplasty, CORR 1925968,1985

[81]. Macquet P: The biomechanics of me Knee and surgical Possibilities of healing osteo Arthritic knee joints. CORR 146:102-110 1980.

[82]. Firestone TP, Krackow KA, Davis JD, Teene SM and Hunger Ford DS: The management of fixed flexion contractures during total Knee arthroplasty CORR 284:221-227, 1992

[83]. Brodley GW, Freeman MAR and Albe Ktsson EJ.Total Prosthetic replacement of akylosed knees. J.Arthroplasty 2: 179-183, 1987

[84]. Agliettie P Windosor R, Buzzi R and Insall jn: Arthroplasty For the stiff or ankylosed knee. J Arthroplasty $4: 1-5,1989$.

[85]. Tew M and Forster IW: Effect of knee replacement on flexion Deformity JBJS 69-B: 395-399, 1987.

[86]. Perry J, Antonelli.D and Ford W: Analysis of knee joint forces During flexed knee stance. JBJS 57A: 961-967, 1975.

[87]. Ritter MA and Camphell ED: Effect of range of motion in the Success of a total knee arthroplasty.J Arthroplasty 2: 95-97,

[88]. Gibbs A, Green G and Taylor J: A comparison of Freeman/Swanson (leett) ad Watlidius prosthesis in total knee Replacement JBJS 61-B: 358-361, 1979.

[89]. Bargen JH, Blaha JD and Freeman MAR: Alignment in total knee arthroplasty, correlated biomechanical and clinical Observations. CORR 173: 178-183, 1983.

[90]. Ewals FC, Jacobs MA, Miegel RE, Walker PS, Poss R and Sledge CB: Kinematic total knee replacement, JBJS 66- A: 1032- 1040, $1 \mathrm{~S} 84$.

[91]. Teeny SM, Krckow KA, Hungerford DS and Jones M: Primary Total knee arthroplasty in patients with severe varus deformity. A Comparative study, CORR 273:19-31,1991.

[92]. Wright RJ, Lima ChBJ, Scott, Thornbill TS: Two to four year Results of posterior cruciate-sparing condylar total knee arthropiasty with uncemented femoral component, CORR 260:80-86, 1990.

[93]. Konig A, Scheidiler M< Rader C and Eulert J: The need for a dual rating System in total knee arthorplasty, CORR 345:161$167,1997$.

[94]. Harvey 1A, Barry K, Kriby SPJ, Johnson R, Elloy MA: Factors affecting the range of movement of total knee arthropiasty, JBJS 75B:950-955, 1993.

[95]. Whiteside LA: The effect of patient age, gender, and tibia Component fixation o pain relief after cement less total knee arthropiasty, CORR 271:21-27. 1990

[96]. Salaffi F, Cavalieri F, Nolli M, Ferraccioli G: Analysis of Disability in knee osteoarthritis, Relationship with age and Psychological variables but not with radiographic scor,. J Rheumatol 18: 15811586,1991. 